\title{
Dynamics of Interacting Neural Networks
}

\author{
W. Kinzel and R. Metzler \\ Institut für Theoretische Physik, Universtität Würzburg, Am Hubland, D-97074 Würzburg, \\ Germany \\ I. Kanter \\ Minerva Center and Department of Physics, Bar Ilan University, 52900 Ramat Gan, Israel
}

(February 15, 2019)

\begin{abstract}
Complex bit sequences generated by a perceptron that learns the opposite of its own prediction are studied, and the success of a student perceptron trained on this sequence is calculated. A system of interacting perceptrons with a directed flow of information is solved analytically. A symmetry breaking phase transition is found with increasing learning rate. A system of interacting perceptrons can develop a good strategy for the problem of adaptive competition known as the minority game.
\end{abstract}

Simple models of neural networks describe a wide variety of phenomena in neurobiology and information theory. Neural networks are systems of elements interacting by adaptive couplings which are trained by a set of examples. After training they function as content addressable associative memory, as classifiers or as prediction algorithms. Using methods of statistical physics many of these phenomena have been elucidated analytically for infinitely large single neural networks [1].

Many phenomena in biology, social science and computer science may be modelled by a system of interacting adaptive algorithms. One of the simplest of such models is a system of interacting perceptrons.

However, nothing is known about general properties of such systems. In this Letter we present analytic solutions of a system of interacting perceptrons. Firstly, we study the behaviour of a self-interacting perceptron generating a bit sequence. Another perceptron which is trained on this sequence has a higher prediction error than for a random bit sequence. Secondly, a set of perceptrons learning from each other by a directed flow of information relaxes to a state of symmetric mutual overlaps. For normalized weight vectors of the perceptrons we find a sharp transition to a state with nonsymmetric mutual overlaps as a function of learning rate. Thirdly, it is shown that a system of interacting perceptrons can develop a 
good strategy for a simple model of adaptive competition in closed markets, the minority game [2,3].

The perceptron is the simplest model of a neural network. It has one layer of synaptic weights $\mathbf{w}=\left(w_{1}, \ldots, w_{M}\right)$ and one output bit $\sigma$ which is given by

$$
\sigma=\operatorname{sign} \sum_{i=1}^{M} w_{i} x_{i}=\operatorname{sign}(\mathbf{w} \cdot \mathbf{x})
$$

$\mathbf{x}$ is the input vector of dimension $M$; for instance it is given by a window of a bit sequence $S_{t} \in+1,-1, t=1,2, \ldots, M$, with $\mathbf{x}_{i}=\left(S_{t-M+1}, \ldots, S_{t}\right)$, or it consists of random binary or gaussian variables. A training example is a pair of input vector and output bit $(\mathbf{x}, \sigma)$; a perceptron learns this example by adjusting its weights to it. Here we consider three well-known learning rules [1]:

$H$ : Hebbian learning

$$
\mathbf{w}_{\text {new }}=\mathbf{w}_{\text {old }}+\frac{\eta}{M} \sigma \mathbf{x}
$$

$P$ : Perceptron learning: $H$ is applied only if the example is misclassified, $\mathbf{w}_{\text {old }} \cdot \mathbf{x} \sigma<0$. $P N$ : Learning with normalization: After each step $P$ the weights are normalized, $\mathbf{w}_{\text {new }}$. $\mathbf{w}_{\text {new }}=1$.

$\eta$ is the size of the learning step which becomes a learning rate in the limit of $M \rightarrow \infty$. In this Letter we study a system of $N$ perceptrons with weight vectors $\mathbf{w}^{1}, \mathbf{w}^{2}, \ldots \mathbf{w}^{N}$ which are trained by a common input vector $\mathbf{x}$ and their mutual output bits $\sigma^{1}, \ldots, \sigma^{N}$.

The first problem we investigate is related to time series prediction. A perceptron which is trained on a bit sequence $S_{1}, S_{2}, \ldots, S_{t}$ is able to make predictions for the next bit $S_{t+1}$ given the window $\mathbf{x}_{t}=\left(S_{t-M+1}, \ldots, S_{t}\right)$ 《t.

Recently it has been emphasized that for any prediction algorithm there exists a bit sequence for which the algorithm completely fails, i.e. the prediction error is $100 \%$ [5]. This sequence is easily constructed: Just take the bit opposite to the prediction. For a perceptron this means that at each time step the network learns the opposite of its own output bit:

$$
\begin{aligned}
S_{t+1} & =-\operatorname{sign}\left(\mathbf{w}_{t} \cdot \mathbf{x}_{t}\right) \\
\mathbf{w}_{t+1} & =\mathbf{w}_{t}+\frac{\eta}{M} S_{t+1} \mathbf{x}_{t} .
\end{aligned}
$$

Note that in this case the rules $H$ and $P$ are identical, at each step the weights are adjusted. For given initial conditions $\mathbf{w}_{0}, \mathbf{x}_{0}$ this algorithm generates an infinite sequence $\left(S_{t}, \mathbf{w}_{t}\right)$.

Our numerical simulations show that such a perceptron generates a rather complex bit sequence $\left(S_{t}\right)$, which after a long transient relaxes to a cycle of length $L>2 M$. Both the average length of the transient and of the cycle increase exponentially with the network size $M$. Details will be published elsewhere. 
Now a second perceptron $\mathbf{w}^{S}$ with different initial conditions is trained on this complex bit sequence using rule $P$. We find that the student network obtains nonzero positive overlap with the bit generator and still has a predicition error of about $75 \%$, larger than the $50 \%$ from a random bit sequence.

In order to perform an analytic calculation, we approximate the input vector $\mathbf{x}_{t}$ from the bit sequence by a random vector $\mathbf{x}$. We obtain the well-known scenario for on-line training which can be solved analytically in the limit $M \rightarrow \infty$ [7,8]. In agreement with Ref. [5] we find that the perceptrons relax to hyperspheres with radii $|\mathbf{w}|=\sqrt{\pi / 8} \eta$ and $\left|\mathbf{w}^{S}\right| \doteq 0.552 \eta$. The angle $\theta$ between generator $\mathbf{w}$ and student $\mathbf{w}^{S}$, which determines the prediction error [6], takes the value of $\theta \doteq 0.706$. This corresponds to a prediction error of $77 \%$, independent of the learning rate $\eta$.

For rule $H$, the sum $\mathbf{w}+\mathbf{w}^{S}$ is not changed by the algorithm. In this case the prediction error depends on $\eta$, but it is always larger that $50 \%$.

These analytical results are in agreement with our simulations for the bit sequence, only the numerical values are slightly different.

In the next scenario we extend up the student-teacher relation to a mutual interaction between the perceptrons: we consider a set of $N$ interacting perceptrons with a directed cyclic flow of information. At each training step all of the networks receive the same randomly chosen input vector $\mathbf{x}$. Now perceptron $\mathbf{w}^{1}$ learn the output from $\mathbf{w}^{2}$, perceptron $\mathbf{w}^{2}$ learns from $\mathbf{w}^{3}, \ldots$, perceptron $\mathbf{w}^{N}$ learns from perceptron $\mathbf{w}^{1}$. Our analytical and numerical calculations give the following result: Starting from random initial weight vectors with length $w_{0}=\left|\mathbf{w}_{0}\right|$ and using perceptron learning rule $P$ for each of the networks, the system runs into a state of complete symmetry with identical overlaps $\mathbf{w}^{i} \cdot \mathbf{w}^{j}$ for all pairs $(i, j)$. The stationary state is given by the equation

$$
\eta \theta \sqrt{1+(N-1) \cos \theta}=\sqrt{2 \pi} w_{0}(1-\cos \theta) .
$$

Fig. [ 1 shows the result. For small learning rate all perceptrons agree with each other, their mutual angle $\theta$ is close to zero. With increasing learning rate the angle increases to its maximal possible value. The sum of the weight vectors $\sum_{i=1}^{N} \mathbf{w}^{i}$ is constant under this learning rule, because for every perceptron that learns the pattern with a positive sign there is a subsequent neighbour that learns it with a negative sign. For $\eta \rightarrow \infty$ the norm of this sum is negligible compared to $\left|\mathbf{w}^{i}\right|$, and the vectors form a hypertetrahedron which gives $\cos \theta=-1 /(N-1)$. Note that the final stationary state has a higher symmetry than the ring flow of information.

The effective repulsion between the weight vectors can be understood geometrically in the case of two perceptrons: the sum $\mathbf{w}^{1}+\mathbf{w}^{2}$ is conserved; the fixed point results from an equilibrium between learning the component of $\mathbf{x}$ parallel to the $\mathbf{w}^{1}-\mathbf{w}^{2}$-plane (which decreases $\theta$ ) and the component perpendicular to this plane (which increases $\theta$ ). 
The symmetric behaviour turns out to be different with learning rule $P N$, where all the weight vectors $\mathbf{w}^{i}$ remain on a sphere $\left|\mathbf{w}^{i}\right|=1$. For small learning rate the system runs into a symmetric state given by

$$
\eta \theta=\sqrt{2 \pi}(1-\cos \theta)
$$

(compare to Eqn. ॠ). However, this equation can only be geometrically realized up to a critical value $\eta_{c}(N)$, where the hypertetrahedron configuration is reached and the sum of the $\mathbf{w}^{i}$ vanishes; there is also a maximal $\eta_{c} \doteq 1.82$, above which no solution of Eqn.5 exists. For larger learning rates $\eta>\eta_{c}(N)$ our numerical simulations give the following results as shown in Fig. 2:

- For $N=3$ the state remains in the triangular configuration $\cos \theta=-1 / 2$.

- For odd values $N \geq 5$ the high symmetry is broken spontaneously. However, the symmetry of the ring is still conserved. This means, for instance, that for $N=7, \theta_{13}$ is the same as $\theta_{24}$ and $\theta_{35}$, but there are three different values of mutual angles $\theta_{12}, \theta_{13}$ and $\theta_{14}$.

- For $N=2$ there is a discontinuous transition to an antiparallel state.

- For even values $N \geq 4$ the scenario agrees with the corresponding one for odd values. However, for higher learning rates we observe an additional discontinuous transition to pairing: Two subsets are formed with antiparallel alignment between the subsets.

Hence, with increasing learning rate the symmetry of the system of interacting perceptrons is broken, but the state still has the symmetry of the ring.

Finally we show that a system of interacting networks can solve a problem of adaptive competition which was recently introduced by Challet and Zhang [2]. It is a model of a closed market where $N$ agents are competing for limited resources and where the individual profit depends on the action of the whole community.

The model consists of $N$ agents who at each time step have to choose between actions $\sigma^{i}=+1$ or $\sigma^{i}=-1, i=1, \ldots, N$. The profit of each agent depends on the minority decision; each agent gains $g^{i}=+1$ if he belongs to the minority, and he pays +1 if he belongs to the majority of the common decision. Hence, one has $g^{i}=-\sigma^{i} \operatorname{sign}\left(\sum_{j=1}^{N} \sigma^{j}\right)$. The global profit is given by

$$
G=\sum_{i=1}^{N} g^{i}=-\left|\sum_{j=1}^{N} \sigma^{j}\right|<0,
$$

the cashier always makes profit. Now each agent uses an algorithm which should maximize his profit. In this model agents know only the history of the minority sign $S_{t}=$ 
$-\operatorname{sign}\left(\sum_{j=1}^{N} \sigma_{t}^{j}\right)$ for each previous time step $t$, and the agents are not allowed to exchange informations.

If each agent makes a random decision $\sigma_{i}$, the mean square global loss is

$$
\left\langle G^{2}\right\rangle=N
$$

It is very hard to find an algorithm which performs better than (6). Previous investigations study algorithms where each agent has two random tables for the $2^{M}$ possible histories $\mathbf{x}_{t}=\left(S_{t-M+1}, \ldots S_{t}\right)$. Each table receives a score, and the one with the larger score is chosen. Here we use a perceptron for each agent to make the decision. At each time its decision is $\sigma_{i}=\operatorname{sign}\left(\mathbf{w}^{i} \cdot \mathbf{x}\right)$ and each perceptron is trained by the minority decision $S_{t}$,

$$
\mathbf{w}_{t+1}^{i}=\mathbf{w}_{t}^{i}-\frac{\eta}{M} \mathbf{x}_{t} \operatorname{sign}\left(\sum_{j=1}^{N} \operatorname{sign}\left(\mathbf{x} \cdot \mathbf{w}^{j}\right)\right) .
$$

Hence, the bit sequence $\left(S_{t}\right)$ is generated by the negative output of a committee machine. Each weight vector is changed by the same increment, hence only the center of mass of the weight vectors changes during the learning process.

Approximating the input $\mathbf{x}$ by a random one, we derived the equation of motion of the norm of the center of mass; the fixed point describes the global gain in the long run. To simplify the calculation, the initial norms $\left|\mathbf{w}_{0}^{i}\right|$ are set to 1 , the sum $\sum_{i}^{N} \mathbf{w}_{0}^{i}$ is 0 , and the scalar products are symmetric: $\mathbf{w}_{0}^{i} \cdot \mathbf{w}_{0}^{j}=-1 /(N-1)$ for $i \neq j$. From the calculation we obtain

$$
\begin{aligned}
\left\langle G^{2}\right\rangle / N & =1+(N-1)\left(1-\frac{2}{\pi} \arccos \frac{A-1 /(N-1)}{A+1}\right) \\
A & =(N-1) \frac{\pi \eta^{2}}{16}\left(1+\sqrt{1+\frac{16}{\pi N \eta^{2}}}\right) .
\end{aligned}
$$

This equation agrees with simulations of both the real time series and random patterns, as shown in Fig. 3. Very similar results (up to factors of $1+1 / \sqrt{N}$ ) are found analytically and in simulations by starting with uncorrelated random vectors. For small learning rate $\eta \rightarrow 0$ we obtain the best global gain

$$
\left\langle G^{2}\right\rangle=\left(1-\frac{2}{\pi}\right) N \simeq 0.363 N
$$

Hence, the system of interacting networks performs better than the random decision. In fact, there are several advantages of the system of neural networks compared to the algorithm of scoring random tables.

Firstly, the size $M$ of the history does not have to be adjusted to the number of agents in order to perform better than random. Our analysis implicitly assumes that $M \leq N-1$ 
and both $M$ and $N$ are large, but simulations show good qualitative agreement even for $N=21, M=4$. For small $M,\left\langle G^{2}\right\rangle / N$ even tends to be smaller than predicted for $M=\infty$.

Secondly, on average all of the agents perform identically. There is no phase transition between a set of successful agents and losers, as found in Ref. [3] for the random tables. This is clear from the geometrical interpretion: similar to the bit generator studied above, the center of mass does a random walk on a hypersphere around the origin. The radius depends on the learning rate; if the radius is smaller than $\sqrt{N}$ (obtained from when adding up $N$ random vectors of norm 1), the "strategies" are distributed better than random. As the center of mass moves, each perceptron shifts from the current majority side to the minority side and back.

It would be interesting to study other network architectures and learning rules to see whether the profit of a system of competing neural networks can still be improved.

This work benefitted from a seminar at the Max-Planck Institut für Physik komplexer Systeme, Dresden. The authors want to thank Michael Biehl and Georg Reents for useful discussions, and Andreas Engel and Johannes Berg for their introduction to the minority game. 


\section{REFERENCES}

[1] J. Hertz, A. Krogh, and R.G. Palmer, Introduction to the Theory of Neural Computation (Addison-Wesley, 1991).

[2] D. Challet and Y.-C. Zhang, Physica 246 A, 407 (1997).

[3] R. Savit, R. Manuca, and R.Riolo, Phys. Rev. Lett. 82, 2203 (1999); D. Challet, M. Marsili and R. Zecchina, to be published.

[4] E. Eisenstein, I. Kanter, D. A. Kessler, and W. Kinzel, Phys. Rev. Lett. 74, 6 (1995); a computer demonstration is found in W. Kinzel and G. Reents, Physics by Computer (Springer Verlag 1998).

[5] H. Zhu and W. Kinzel, Neural Computation 10, 2219 (1998).

[6] M. Opper and W. Kinzel, in: Models of Neural Networks III, edited by E. Domany, J.L. van Hemmen and K. Schulten (Springer Verlag 1995), p.151

[7] M. Biehl and H. Schwarze, J. Phys. A 28, 643 (1995).

[8] D. Saad and S. Solla, Phys. Rev. Lett. 74, 4337 (1995). 


\section{FIGURES}

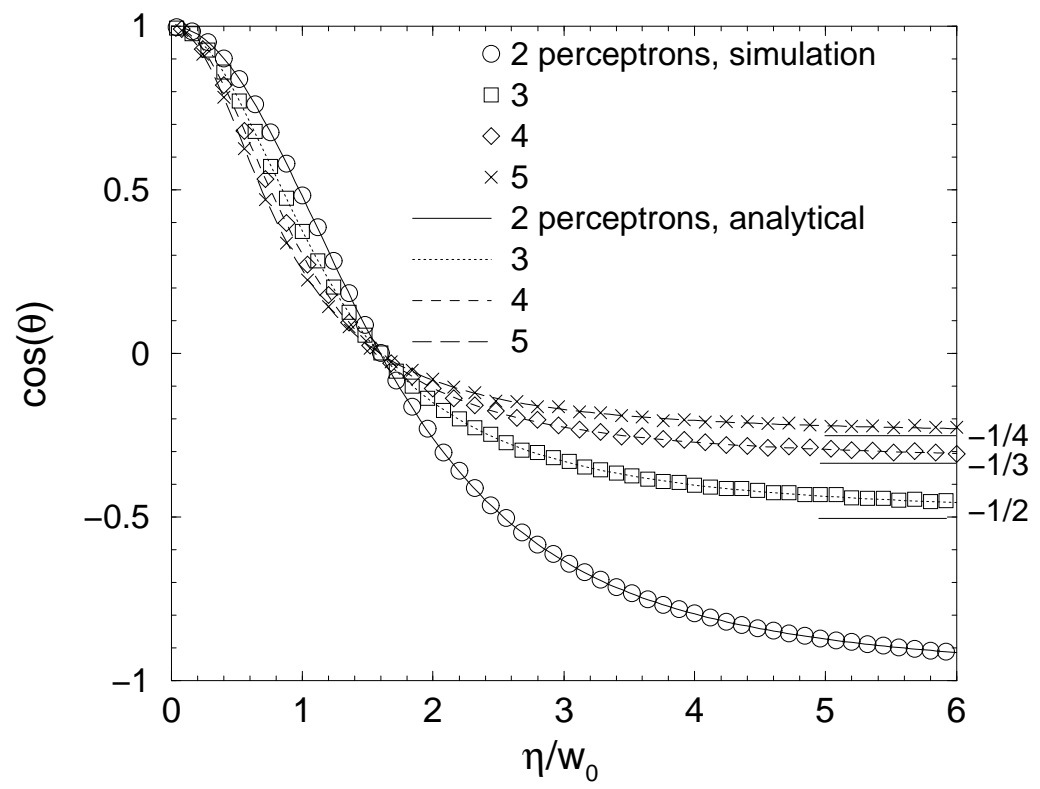

FIG. 1. Fixed point of cyclic learning with alg. $P$ : simulations with $M=100$ for 2 to 5 perceptrons and solutions of Eqn. 1 .
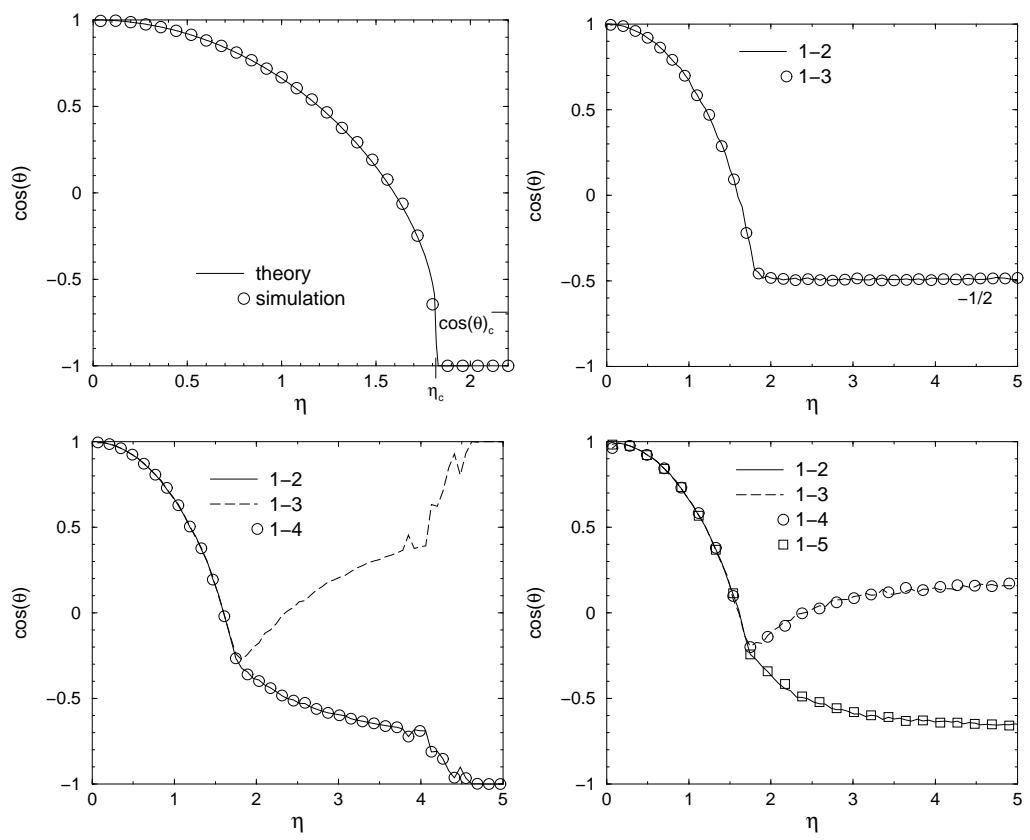

FIG. 2. Fixed points of $\cos \theta$ in cyclic learning using rule $P N$ for $2,3,4$, and 5 perceptrons, respectively. 


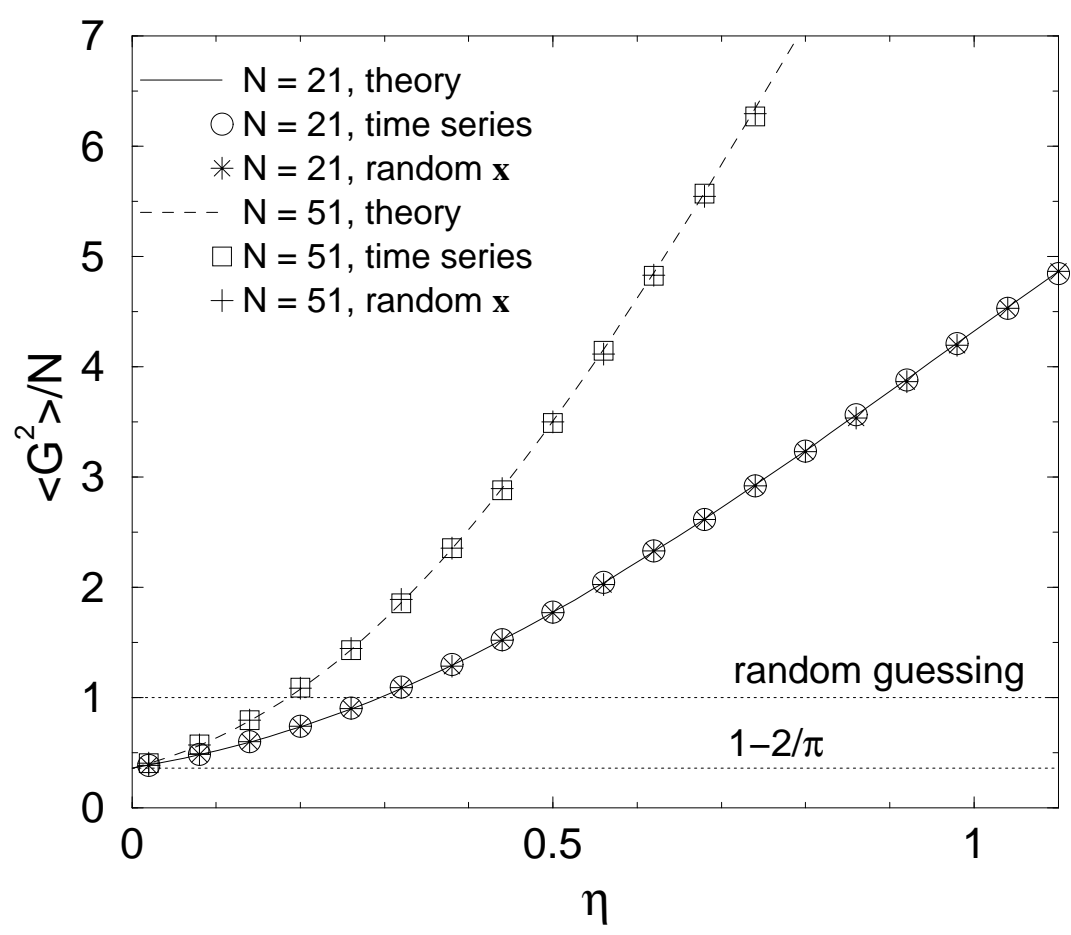

FIG. 3. Average loss $\left\langle G^{2}\right\rangle / N$ versus learning rate in the Minority Game, using learning rule $H$. Simulations used $M=100$. 